\title{
DETERMINANTS CONTAINING POWERS OF POLYNOMIAL SEQUENCES
}

\author{
H.-H. LEUNG
}

Received 25 September, 2018

\begin{abstract}
We derive identities for the determinants of matrices whose entries are (rising) powers of (products of) polynomials that satisfy a recurrence relation. In particular, these results cover the cases for Fibonacci polynomials, Lucas polynomials and certain orthogonal polynomials. These identities naturally generalize the determinant identities obtained by Alfred, Carlitz, Prodinger, Tangboonduangjit and Thanatipanonda.
\end{abstract}

2010 Mathematics Subject Classification: 11B39; 11C20

Keywords: Fibonacci polynomial, Lucas polynomial, Chebyshev polynomial, determinant identity

\section{INTRODUCTION}

Let $\left(F_{n}\right)_{n \geq 0}$ be the Fibonacci sequence. Let $m \geq 1$ and $n$ be any nonnegative integer. Let $\left[F_{n+i+j}^{m}\right]_{0 \leq i, j \leq m}$ be the $(m+1) \times(m+1)$ matrix with entries $F_{n+i+j}$, $0 \leq i, j \leq m$. In 1966, Carlitz [4] derived the following determinant identity for the matrix $\left[F_{n+i+j}^{m}\right]_{0 \leq i, j \leq m}$ :

$$
\operatorname{det}\left(\left[F_{n+i+j}^{m}\right]_{0 \leq i, j \leq m}\right)=(-1)^{(n+1)\left(\begin{array}{c}
m+1 \\
2
\end{array}\right)}\left(F_{1}^{m} F_{2}^{m-1} \cdots F_{m}\right)^{2} \prod_{i=0}^{m}\left(\begin{array}{c}
m \\
i
\end{array}\right) .
$$

This identity is related to the problems posted by Alfred [1, p. 48] in 1963 and Parker [9, p. 303] in 1964 respectively. Let $s$ and $k$ be any integers. Tangboonduangjit and Thanatipanonda [12] generalized the determinant identity (1.1) as follows:

$$
\operatorname{det}\left(\left[F_{s+k(n+i+j)}^{m}\right]_{0 \leq i, j \leq m}\right)=(-1)^{(s+k n+1)\left(\begin{array}{c}
m+1 \\
2
\end{array}\right)}\left(F_{k}^{m} F_{2 k}^{m-1} \cdots F_{m k}\right)^{2} \prod_{i=0}^{m}\left(\begin{array}{c}
m \\
i
\end{array}\right) \text {. }
$$

Let $F_{n}^{\langle m\rangle}$ be the rising powers of the Fibonacci numbers defined by

$$
F_{n}^{\langle m\rangle}:=F_{n} F_{n+1} \cdots F_{n+m-1} .
$$

The author was supported in part by the UAEU Startup Grant 2016, Grant No. G00002235. 
Prodinger [10] obtained the following determinant identity for the matrix $\left[F_{n+i+j}^{\langle m\rangle}\right]_{0 \leq i, j \leq m}$ :

$$
\operatorname{det}\left(\left[F_{n+i+j}^{\langle m\rangle}\right]_{0 \leq i, j \leq m}\right)=(-1)^{n\left(\begin{array}{c}
m+1 \\
2
\end{array}\right)+\left(\begin{array}{c}
m+2 \\
3
\end{array}\right)}\left(F_{1} F_{2} \cdots F_{m}\right)^{m+1} .
$$

Tangboonduangjit and Thanatipanonda [11] generalized the determinant identity (1.3) as follows:

$\operatorname{det}\left(\left[F_{n+i+j}^{\langle m\rangle}\right]_{0 \leq i, j \leq d-1}\right)=(-1)^{n\left(\begin{array}{c}d \\ 2\end{array}\right)+\left({ }^{d+1}\right)} \prod_{i=1}^{d-1}\left(F_{i} F_{m+1-i}\right)^{d-i} \cdot \prod_{i=d-1}^{2(d-1)} F_{n+i}^{\langle m+1-d\rangle}$.

where $d \geq 2$. It is worthwhile to note that Tangboonduangjit and Thanatipanonda $[11,12]$ derived the determinant identities more generally, for matrices whose entries include (rising) powers of terms that satisfy a second-order linear recurrence relation with constant coefficients. By using analogous techniques in determinant calculus, we derive determinant identities for matrices whose entries are (rising) powers of polynomials that satisfy certain recurrence relations. As corollaries, we provide determinant identities for matrices whose entries are (rising) powers of Fibonacci polynomials, Lucas polynomials and certain orthogonal polynomials. As an application, we obtain new identities in the case of Fibonacci numbers. For example, for $n \geq 1$, by Corollary 2, we get

$$
\operatorname{det}\left(\left[\frac{1}{F_{n+i+j}}\right]_{0 \leq i, j \leq m}\right)=\frac{(-1)^{n\left(\begin{array}{c}
(m+1) \\
2
\end{array}\right)} \prod_{i=0}^{m} F_{i+1}^{2(m-i)}}{\prod_{0 \leq i, j \leq m} F_{n+i+j}} .
$$

\section{MAIN RESULTS}

Definition 1. Let $p, q, r, a, b$ and $c$ be any real numbers. Let $\mathbb{Z}_{\geq 0}=\{0,1,2, \ldots\}$. The sequence of polynomials in variable $x$,

$$
\mathcal{P}(x)(p, q, r ; a, b, c):=\left(P_{n}\right)_{n \in \mathbb{Z}},
$$

is defined by

$$
P_{0}:=p, \quad P_{1}:=q x+r, \quad P_{n+2}:=(a x+b) P_{n+1}+c P_{n}, \text { for } n \in \mathbb{Z}_{\geq 0} .
$$

For $n<0, P_{n}$ is defined by

$$
P_{n}:=-\frac{a x+b}{c} P_{n+1}+\frac{1}{c} P_{n+2} .
$$

The discriminant $\Delta_{\mathcal{P}}$ is defined by

$$
\Delta_{\mathcal{P}}:=\left(q^{2}-a p q\right) x^{2}+(2 q r-a p r-b p q) x+\left(r^{2}-b p r-c p^{2}\right) .
$$


Theorem 1. Let $\mathcal{P}(x)\left(p_{1}, q_{1}, r_{1} ; a, b, c\right)=\left(P_{n}\right)_{n \in \mathbb{Z},}, \mathcal{Q}(x)\left(p_{2}, q_{2}, r_{2} ; a, b, c\right)=$ $\left(Q_{n}\right)_{n \in \mathbb{Z}}$ and $U(x)(0,0,1 ; a, b, c)=\left(U_{n}\right)_{n \in \mathbb{Z}}$ be the sequences of polynomials defined by real numbers $p_{1}, p_{2}, q_{1}, q_{2}, r_{1}, r_{2}, a, b, c$ where $c \neq 0$. Then

$$
P_{s+i} Q_{s+j}-P_{s} Q_{s+i+j}=(-c)^{s}\left(P_{1} Q_{j}-P_{0} Q_{j+1}\right) U_{i}
$$

for all integers $s, i, j$.

Proof. We prove it by induction on $i$. It is trivial for $i=0$. If $i=1$, we have

$$
\begin{aligned}
\left(\begin{array}{cc}
P_{s+1} & Q_{s+j+1} \\
P_{s} & Q_{s+j}
\end{array}\right) & =\left(\begin{array}{cc}
a x+b & c \\
1 & 0
\end{array}\right)\left(\begin{array}{cc}
P_{s} & Q_{s+j} \\
P_{s-1} & Q_{s+j-1}
\end{array}\right)= \\
& =\left(\begin{array}{cc}
a x+b & c \\
1 & 0
\end{array}\right)^{s}\left(\begin{array}{cc}
P_{1} & Q_{j+1} \\
P_{0} & Q_{j}
\end{array}\right), \\
\left(\begin{array}{cc}
P_{s+1} & Q_{s+j+1} \\
P_{s} & Q_{s+j}
\end{array}\right) & =\left(\begin{array}{cc}
a x+b & c \\
1 & 0
\end{array}\right)^{-1}\left(\begin{array}{cc}
P_{s+2} & Q_{s+j+2} \\
P_{s+1} & Q_{s+j+1}
\end{array}\right)= \\
& =\left(\begin{array}{cc}
a x+b & c \\
1 & 0
\end{array}\right)^{s}\left(\begin{array}{cc}
P_{1} & Q_{j+1} \\
P_{0} & Q_{j}
\end{array}\right)
\end{aligned}
$$

for $s \geq 0$ and $s<0$ respectively. We take the determinants on both sides of (2.1) and (2.2) to get

$$
\begin{aligned}
P_{s+1} Q_{s+j}-P_{s} Q_{s+j+1} & =(-c)^{s}\left(P_{1} Q_{j}-P_{0} Q_{j+1}\right) \\
& =(-c)^{s}\left(P_{1} Q_{j}-P_{0} Q_{j+1}\right) U_{1} .
\end{aligned}
$$

For $i>1$, we assume that the identity is true for $i-1$ and $i-2$. We have

$$
\begin{aligned}
& P_{s+i} Q_{s+j}-P_{s} Q_{s+i+j} \\
& =\left|\begin{array}{cc}
P_{s+i} & Q_{s+i+j} \\
P_{s} & Q_{s+j}
\end{array}\right| \\
& =\left|\begin{array}{cc}
(a x+b) P_{s+i-1}+c P_{s+i-2} & (a x+b) Q_{s+i+j-1}+c Q_{s+i+j-2} \\
P_{s} & Q_{s+j}
\end{array}\right| \\
& =(a x+b)\left|\begin{array}{cc}
P_{s+i-1} & Q_{s+j+(i-1)} \\
P_{s} & Q_{s+j}
\end{array}\right|+c\left|\begin{array}{cc}
P_{s+i-2} & Q_{s+j+(i-2)} \\
P_{s} & Q_{s+j}
\end{array}\right| \\
& =(a x+b)(-c)^{s}\left(P_{1} Q_{j}-P_{0} Q_{j+1}\right) U_{i-1}+c(-c)^{s}\left(P_{1} Q_{j}-P_{0} Q_{j+1}\right) U_{i-2} \\
& =(-c)^{s}\left(P_{1} Q_{j}-P_{0} Q_{j+1}\right)\left((a x+b) U_{i-1}+c U_{i-2}\right) \\
& =(-c)^{s}\left(P_{1} Q_{j}-P_{0} Q_{j+1}\right) U_{i} .
\end{aligned}
$$

For $i<0$, we assume that the identity is true for $i+1$ and $i+2$. We have

$$
\begin{aligned}
& P_{s+i} Q_{s+j}-P_{s} Q_{s+i+j}=\left|\begin{array}{cc}
P_{s+i} & Q_{s+i+j} \\
P_{s} & Q_{s+j}
\end{array}\right| \\
& =\left|\begin{array}{cc}
-\frac{a x+b}{c} P_{s+i+1}+\frac{1}{c} P_{S+i+2} & -\frac{a x+b}{c} Q_{s+i+j+1}+\frac{1}{c} Q_{s+i+j+2} \\
P_{S} & Q_{s+j}
\end{array}\right|
\end{aligned}
$$




$$
\begin{aligned}
& =-\frac{a x+b}{c}\left|\begin{array}{cc}
P_{s+i+1} & Q_{s+i+j+1} \\
P_{s} & Q_{s+j}
\end{array}\right|+\frac{1}{c}\left|\begin{array}{cc}
P_{s+i+2} & Q_{s+i+j+2} \\
P_{s} & Q_{s+j}
\end{array}\right| \\
& =-\frac{a x+b}{c}(-c)^{s}\left(P_{1} Q_{j}-P_{0} Q_{j+1}\right) U_{i+1}+\frac{1}{c}(-c)^{s}\left(P_{1} Q_{j}-P_{0} Q_{j+1}\right) U_{i+2} \\
& =(-c)^{s}\left(P_{1} Q_{j}-P_{0} Q_{j+1}\right)\left(-\frac{a x+b}{c} U_{i+1}+\frac{1}{c} U_{i+2}\right) \\
& =(-c)^{s}\left(P_{1} Q_{j}-P_{0} Q_{j+1}\right) U_{i} .
\end{aligned}
$$

Remark 1. We recover the generalized Catalan Identity by Melham and Shannon [8] (see also Tangboonduangjit and Thanatipanonda [12, Proposition 1]) by substituting $x=1$ in Theorem 1 .

Corollary 1. Let $\mathcal{P}(x)(p, q, r ; a, b, c)=\left(P_{n}\right)_{n \in \mathbb{Z}}$ and $U(x)(0,0,1 ; a, b, c)=$ $\left(U_{n}\right)_{n \in \mathbb{Z}}$ be the sequences of polynomials defined by real numbers $p, q, r, a, b, c$ where $c \neq 0$. Then

$$
\begin{aligned}
P_{j} P_{1}-P_{0} P_{j+1} & =\Delta_{\mathcal{P}} \cdot U_{j}, \\
P_{s+i} P_{s+j}-P_{S} P_{s+i+j} & =(-c)^{s} \Delta_{\mathcal{P}} \cdot U_{i} U_{j}
\end{aligned}
$$

where $\Delta_{\mathcal{P}}$ is the discriminant of $\mathcal{P}(x)(p, q, r ; a, b, c)$.

Proof. By setting $s=0, j=1, p_{1}=p_{2}=p, q_{1}=q_{2}=q, r_{1}=r_{2}=r$ in Theorem 1 , we get

$$
P_{i} P_{1}-P_{0} P_{i+1}=\left(P_{1} P_{1}-P_{0} P_{2}\right) U_{i} .
$$

We note that $P_{0}=p, P_{1}=q x+r$ and $P_{2}=(a x+b) P_{1}+c P_{0}=(a x+b)(q x+$ $r)+c p$. Hence, we obtain (2.4) by simplifying (2.6). On the other hand, by setting $p_{1}=p_{2}=p, q_{1}=q_{2}=q, r_{1}=r_{2}=r$ in Theorem 1 , we get

$$
P_{s+i} P_{s+j}-P_{s} P_{s+i+j}=(-c)^{s}\left(P_{1} P_{j}-P_{0} P_{j+1}\right) U_{i}=(-c)^{s} \Delta_{\mathcal{P}} \cdot U_{i} U_{j}
$$

in which the last equality is based on (2.4).

Lemma 1. Let $m \geq 1$. Let $B_{i}, D_{i}$ be polynomials in variable $x, A_{i}, C_{i}$ be rational functions in variable $x$, for $0 \leq i \leq m$. Let $\left[\left(A_{j} B_{i}+C_{j} D_{i}\right)^{m}\right]_{0 \leq i, j \leq m}$ be the $(m+$ $1) \times(m+1)$ matrix with entries $\left(A_{j} B_{i}+C_{j} D_{i}\right)^{m}, 0 \leq i, j \leq m$. Then we have the following determinant identity:

$$
\begin{aligned}
& \operatorname{det}\left(\left[\left(A_{j} B_{i}+C_{j} D_{i}\right)^{m}\right]_{0 \leq i, j \leq m}\right)= \\
& =\prod_{0 \leq i<j \leq m}\left(\left(B_{i} D_{j}-B_{j} D_{i}\right)\left(A_{i} C_{j}-A_{j} C_{i}\right)\right) \cdot \prod_{i=0}^{m}\left(\begin{array}{c}
m \\
i
\end{array}\right) .
\end{aligned}
$$


Proof. We invoke the following result by Krattenthaler [7, Lemma 10] (see also Tangboonduangjit and Thanatipanonda [12, Lemma 3]:

$$
\operatorname{det}\left(\left[\left(c_{j} d_{i}+1\right)^{m}\right]_{0 \leq i, j \leq m}\right)=\prod_{0 \leq i<j \leq m}\left(\left(d_{i}-d_{j}\right)\left(c_{i}-c_{j}\right)\right) \cdot \prod_{i=0}^{m}\left(\begin{array}{c}
m \\
i
\end{array}\right)
$$

where $c_{j}, d_{i}$ are real numbers for $0 \leq i, j \leq m$. First, we prove the lemma for polynomials $A_{i}, B_{i}, C_{i}, D_{i}$ for all $0 \leq i \leq m$. For the values of $x$ such that $C_{j} \neq 0$ and $D_{i} \neq 0$ for $0 \leq i, j \leq m$, let

$$
c_{j}=\frac{A_{j}}{C_{j}}, \quad d_{i}=\frac{B_{i}}{D_{i}}, \text { for } 0 \leq i, j \leq m
$$

We note that

$$
\begin{aligned}
& \operatorname{det}\left(\left[\left(c_{j} d_{i}+1\right)^{m}\right]_{0 \leq i, j \leq m}\right)=\operatorname{det}\left(\left[\left(\frac{A_{j} B_{i}+C_{j} D_{i}}{C_{j} D_{i}}\right)^{m}\right]_{0 \leq i, j \leq m}\right) \\
& =\left(\prod_{0 \leq i \leq m} \frac{1}{\left(C_{i} D_{i}\right)^{m}}\right) \cdot \operatorname{det}\left(\left[\left(A_{j} B_{i}+C_{j} D_{i}\right)^{m}\right]_{0 \leq i, j \leq m}\right) .
\end{aligned}
$$

Also, we have

$$
\begin{aligned}
& \prod_{0 \leq i<j \leq m}\left(\left(d_{i}-d_{j}\right)\left(c_{i}-c_{j}\right)\right)=\prod_{0 \leq i<j \leq m}\left(\left(\frac{B_{i} D_{j}-B_{j} D_{i}}{D_{i} D_{j}}\right) \cdot\left(\frac{A_{i} C_{j}-A_{j} C_{i}}{C_{i} C_{j}}\right)\right) \\
= & \prod_{0 \leq i \leq m}\left(\frac{1}{\left(C_{i} D_{i}\right)^{m}}\right) \cdot \prod_{0 \leq i<j \leq m}\left(\left(B_{i} D_{j}-B_{j} D_{i}\right)\left(A_{i} C_{j}-A_{j} C_{i}\right)\right) .
\end{aligned}
$$

By (2.8), (2.9), (2.10), we get (2.7) as desired.

Based on the facts that there are only a finite number of roots for $C_{j}, D_{i}$ where $0 \leq i, j \leq m$ and the determinant of a matrix with polynomial entries is a continuous function in $x$, the equality (2.7) still holds true for the values of $x$ such that $C_{j}=0$ or $D_{i}=0$ for some $i$ or $j$.

Next, we assume that $A_{i}$ and $C_{i}$ are rational functions for all $0 \leq i \leq m$. We write $A_{i}$ and $C_{i}$ as follows:

$$
A_{i}=\frac{E_{i}}{F_{i}}, \quad C_{i}=\frac{G_{i}}{H_{i}} \text { for } 0 \leq i \leq m,
$$


where $E_{i}, F_{i}, G_{i}, H_{i}$ are all polynomials for $0 \leq i \leq m$. For the values of $x$ such that $F_{i} \neq 0$ and $H_{i} \neq 0$ for all $0 \leq i \leq m$, we get

$$
\begin{aligned}
& \operatorname{det}\left(\left[\left(A_{j} B_{i}+C_{j} D_{i}\right)^{m}\right]_{0 \leq i, j \leq m}\right)=\operatorname{det}\left(\left[\left(\frac{H_{j} E_{j} B_{i}+D_{i} F_{j} G_{j}}{F_{j} H_{j}}\right)^{m}\right]_{0 \leq i, j \leq m}\right) \\
= & \left(\prod_{0 \leq i \leq m} \frac{1}{\left(F_{i} H_{i}\right)^{m}}\right) \cdot \operatorname{det}\left(\left[\left(\left(H_{j} E_{j}\right) B_{i}+\left(G_{j} F_{j}\right) D_{i}\right)^{m}\right]_{0 \leq i, j \leq m}\right) \\
= & \left(\prod_{0 \leq i \leq m} \frac{1}{\left(F_{i} H_{i}\right)^{m}}\right)\left(\prod_{i=0}^{m}\left(\begin{array}{c}
m \\
i
\end{array}\right)\right) . \\
& \cdot\left(\prod_{0 \leq i<j \leq m}\left(B_{i} D_{j}-B_{j} D_{i}\right)\left(H_{i} E_{i} G_{j} F_{j}-H_{j} E_{j} G_{i} F_{i}\right)\right) \\
= & \left(\prod_{i=0}^{m}\left(\begin{array}{c}
m \\
i
\end{array}\right)\right)\left(\prod_{0 \leq i<j \leq m}\left(B_{i} D_{j}-B_{j} D_{i}\right)\left(\frac{E_{i}}{F_{i}} \cdot \frac{G_{j}}{H_{j}}-\frac{E_{j}}{F_{j}} \cdot \frac{G_{i}}{H_{i}}\right)\right) \\
= & \left(\prod_{i=0}^{m}\left(\begin{array}{c}
m \\
i
\end{array}\right)\right)\left(\prod_{0 \leq i<j \leq m}\left(B_{i} D_{j}-B_{j} D_{i}\right)\left(A_{i} C_{j}-A_{j} C_{i}\right)\right) .
\end{aligned}
$$

For the values of $x$ such that $F_{i}=0$ or $H_{i}=0$ for some $i$, the equality still holds true as the determinant of a matrix with polynomial entries is a continuous function.

Theorem 2. Let $s, k, n$ be any integers, $m \geq 1$. Let $\mathcal{P}(x)(p, q, r ; a, b, c)=\left(P_{n}\right)_{n \in \mathbb{Z}}$ and $\mathcal{U}(x)(0,0,1 ; a, b, c)=\left(U_{n}\right)_{n \in \mathbb{Z}}$ be the sequences of polynomials defined by real numbers $p, q, r, a, b, c$ where $c \neq 0 . \quad$ The determinant of the matrix $\left[P_{s+k(n+i+j)}^{m}\right]_{0 \leq i, j \leq m}$ is given by

$$
\begin{aligned}
& \operatorname{det}\left(\left[P_{s+k(n+i+j)}^{m}\right]_{0 \leq i, j \leq m}\right) \\
& =(-1)^{(s+k n+1)\left(\begin{array}{c}
m+1 \\
2
\end{array}\right)} \Delta_{\mathcal{P}}^{\left(\begin{array}{c}
m+1 \\
2
\end{array}\right)} \cdot c^{(s+k n)\left(\begin{array}{c}
m+1 \\
2
\end{array}\right)+2 k\left(\begin{array}{c}
m+1 \\
3
\end{array}\right)} \prod_{i=0}^{m}\left(\left(\begin{array}{c}
m \\
i
\end{array}\right) U_{k(i+1)}^{2(m-i)}\right)
\end{aligned}
$$

where $\Delta_{\mathcal{P}}$ is the discriminant of $\mathcal{P}(x)(p, q, r ; a, b, c)$.

Proof. By substituting $s=-k m, i=k j^{\prime}, j=s^{\prime}+k\left(n+m+i^{\prime}\right)$ into (2.5) in Corollary 1 and then replacing $s^{\prime}, i^{\prime}, j^{\prime}$ by $s, i, j$ respectively, we get

$$
\begin{aligned}
& P_{k(j-m)} P_{s+k(n+i)}-P_{-k m} P_{s+k(n+i+j)}=(-c)^{-k m} \Delta_{\mathcal{P}} \cdot U_{k j} U_{s+k(n+m+i)}, \\
& P_{s+k(n+i+j)}=\frac{P_{k(j-m)}}{P_{-k m}} \cdot P_{s+k(n+i)}+\frac{-(-c)^{-k m} \Delta_{\mathcal{P}} \cdot U_{k j}}{P_{-k m}} \cdot U_{s+k(n+m+i)} .
\end{aligned}
$$


By substituting $s=s^{\prime}+k\left(n+m+i^{\prime}\right), i=k\left(j^{\prime}-i^{\prime}\right), j=-k m$ into Theorem 1 and then replacing $s^{\prime}, i^{\prime}, j^{\prime}$ by $s, i, j$ respectively, we get

$$
\begin{aligned}
& P_{s+k(n+i)} U_{s+k(n+m+j)}-P_{s+k(n+j)} U_{s+k(n+m+i)} \\
& =(-c)^{s+k(n+m+i)}\left(U_{1} P_{-k m}-U_{0} P_{-k m+1}\right) U_{k(j-i)} \\
& =(-c)^{s+k(n+m+i)} P_{-k m} U_{k(j-i)} .
\end{aligned}
$$

By substituting $s=k i^{\prime}, i=k\left(j^{\prime}-i^{\prime}\right), j=-k m$ into Theorem 1 and then replacing $i^{\prime}, j^{\prime}$ by $i, j$ respectively, we get

$$
P_{k(i-m)} U_{k j}-P_{k(j-m)} U_{k i}=(-c)^{k i} P_{-k m} U_{k(j-i)} .
$$

By (2.11), we get

$$
\begin{aligned}
& \operatorname{det}\left(\left[P_{s+k(n+i+j)}^{m}\right]_{0 \leq i, j \leq m}\right) \\
& =\operatorname{det}\left(\left[\left(\frac{P_{k(j-m)}}{P_{-k m}} \cdot P_{s+k(n+i)}+\frac{-(-c)^{-k m} \Delta_{\mathcal{P}} \cdot U_{k j}}{P_{-k m}} \cdot U_{s+k(n+m+i)}\right)^{m}\right]_{0 \leq i, j \leq m}\right) .
\end{aligned}
$$

By (2.7), the term in (2.14) becomes

$$
\begin{array}{r}
\prod_{i=0}^{m}\left(\begin{array}{c}
m \\
i
\end{array}\right) \cdot \prod_{0 \leq i<j \leq m}\left(\left(P_{s+k(n+i)} U_{s+k(n+m+j)}-P_{s+k(n+j)} U_{s+k(n+m+i)}\right)\right) . \\
\left.\left(\frac{-(-c)^{-k m} \Delta_{\mathcal{P}}}{P_{-k m}^{2}}\left(P_{k(i-m)} U_{k j}-P_{k(j-m)} U_{k i}\right)\right)\right) .
\end{array}
$$

By (2.12), (2.13), the term in (2.15) becomes

$$
\prod_{i=0}^{m}\left(\begin{array}{c}
m \\
i
\end{array}\right) \cdot \prod_{0 \leq i<j \leq m}\left((-1)^{s+k n+1} c^{s+k(n+2 i)} \Delta_{\mathcal{P}} \cdot U_{k(j-i)}^{2}\right) .
$$

As a consequence, we get the desired result by standard counting arguments.

Remark 2. We recover Theorem 5 in the work of Tangboonduangjit and Thanatipanonda [12] by substituting $x=1$ in Theorem 2 .

Next, we look at other determinant identities.

Lemma 2. Let $m \geq 1$. Let $B_{i}, D_{i}$ be polynomials in variable $x$ for $0 \leq i \leq m$. Let $A_{j}, C_{j}$ be rational functions in variable $x$ for $i \in \mathbb{Z}$. Let $\left(d_{i}\right)_{1 \leq i \leq r}$ and $\left(e_{i}\right)_{1 \leq i \leq r}$ be sequences of integers. Then

$$
\operatorname{det}\left(\left[\prod_{f=j+1}^{m}\left(A_{d_{f}} B_{i}+C_{d_{f}} D_{i}\right) \cdot \prod_{g=1}^{j}\left(A_{e_{g}} B_{i}+C_{e_{g}} D_{i}\right)\right]_{0 \leq i, j \leq m}\right)
$$




$$
=\prod_{0 \leq i<j \leq m}\left(B_{i} D_{j}-B_{j} D_{i}\right) \cdot \prod_{1 \leq i \leq j \leq m}\left(C_{e_{i}} A_{d_{j}}-A_{e_{i}} C_{d_{j}}\right) .
$$

Proof. By the factorization method of Krattenthaler [7, Section 4], it is plain to get the following identity:

$$
\begin{aligned}
\operatorname{det}\left(\left[\prod_{f=j+1}^{m}\left(X_{i}+F_{f}\right) \cdot \prod_{g=1}^{j}\left(X_{i}+G_{g}\right)\right]_{0 \leq i, j \leq m}\right) & \\
& =\prod_{0 \leq i<j \leq m}\left(X_{j}-X_{i}\right) \cdot \prod_{1 \leq i \leq j \leq m}\left(F_{j}-G_{i}\right)
\end{aligned}
$$

where $X_{i}$ for $0 \leq i \leq m, D_{j}, E_{j}$ for $1 \leq j \leq m$ are some indeterminates. For the values of $x$ such that $D_{i} \neq 0$ and $A_{j} \neq 0$ for $0 \leq i \leq m$ and $j \in \mathbb{Z}$, let

$$
X_{i}=\frac{B_{i}}{D_{i}}, \quad F_{j}=\frac{C_{d_{j}}}{A_{d_{j}}}, \quad G_{j}=\frac{C_{e_{j}}}{A_{e_{j}}}
$$

for $0 \leq i \leq m$ and $1 \leq j \leq m$. By similar reasoning as in the proof of Lemma 1 , we get the desired result by clearing the denominators on both sides of (2.17). For the values of $x$ which are the roots of $D_{i}$ or $A_{j}$ for some $i$ or $j$, the equality still holds true based on the fact that the determinant of a matrix with polynomial entries is a continuous function.

Theorem 3. Let $s, k, n$ be any integers, $m \geq 1$. Let $\mathcal{P}(x)(p, q, r ; a, b, c)=\left(P_{n}\right)_{n \in \mathbb{Z}}$ and $U(x)(0,0,1 ; a, b, c)=\left(U_{n}\right)_{n \in \mathbb{Z}}$ be the sequences of polynomials defined by real numbers $p, q, r, a, b, c$ where $c \neq 0$. Let $\left(d_{i}\right)_{1 \leq i \leq m}$ and $\left(e_{i}\right)_{1 \leq i \leq m}$ be sequences of integers. Then

$$
\begin{aligned}
& \operatorname{det}\left(\left[\prod_{f=j+1}^{m} P_{s+k\left(n+i+d_{f}\right)} \prod_{g=1}^{j} P_{s+k\left(n+i+e_{g}\right)}\right]_{0 \leq i, j \leq m}\right) \\
& =\left(-\Delta_{\mathcal{P}}\right)^{\left(\begin{array}{c}
m+1 \\
2
\end{array}\right)}(-c)^{(s+k n)\left(\begin{array}{c}
m+1 \\
2
\end{array}\right)+k\left(\begin{array}{c}
m+1 \\
3
\end{array}\right)} \prod_{l=1}^{m} U_{k l}^{m+1-l} \cdot \prod_{1 \leq i \leq j \leq m}(-c)^{k d_{j}} U_{k\left(e_{i}-d_{j}\right)}
\end{aligned}
$$

where $\Delta_{\mathcal{P}}$ is the discriminant of $\mathcal{P}(x)(p, q, r ; a, b, c)$.

Proof. By (2.11), Lemma 2 and Corollary 1, the theorem can be proved in the same way as in the proof the Theorem 2 .

Lemma 3. Let $m \geq 1$. Let $A_{i}, B_{i}$ are polynomials in variable $x$ for $0 \leq i \leq m$. Let $C_{i}, D_{i}$ be rational functions in variable $x$ for $0 \leq i \leq m$. Then,

$$
\operatorname{det}\left(\left[\frac{1}{A_{i} D_{j}+B_{i} C_{j}}\right]_{0 \leq i, j \leq m}\right)=\frac{\prod_{0 \leq i<j \leq m}\left(A_{i} B_{j}-A_{j} B_{i}\right)\left(C_{i} D_{j}-D_{i} C_{j}\right)}{\prod_{0 \leq i, j \leq m}\left(A_{i} D_{j}+B_{i} C_{j}\right)}
$$

provided that the denominators on both sides of the identity are nonzero. 
Proof. First, we invoke a result of Krattenthaler [7, Theorem 12]. That is,

$$
\operatorname{det}\left(\left[\frac{1}{x_{i}+y_{j}}\right]_{0 \leq i, j \leq m}\right)=\frac{\prod_{0 \leq i<j \leq m}\left(x_{i}-x_{j}\right)\left(y_{i}-y_{j}\right)}{\prod_{0 \leq i, j \leq m}\left(x_{i}+y_{j}\right)}
$$

where $x_{i}$ and $y_{i}$ are indeterminates for $0 \leq i, j \leq m$. We first assume that $A_{i}, B_{i}, C_{i}, D_{i}$ are all polynomials for all $0 \leq i \leq m$. For the values of $x$ such that $B_{i}, D_{i}$ are nonzero for all $0 \leq i \leq m$, let

$$
x_{i}=\frac{A_{i}}{B_{i}}, \quad y_{i}=\frac{C_{i}}{D_{i}} \text { for } 0 \leq i \leq m .
$$

By similar reasoning as shown in the proof of Lemma 1, we get the desired result by some algebraic simplification for the cases where $A_{i}, B_{i}, C_{i}, D_{i}$ are polynomials for all $0 \leq i \leq m$.

We extend the proof to the cases where $C_{i}$ and $D_{i}$ are rational functions by the same arguments as in the proof of Lemma 1 , based on the fact that the determinant of a matrix with rational functions as entries is a continuous function provided that the denominators on both sides of the identity are nonzero.

Theorem 4. Let $s, k, n$ be any integers, $m \geq 1$. Let $\mathcal{P}(x)(p, q, r ; a, b, c)=\left(P_{n}\right)_{n \in \mathbb{Z}}$ and $U(x)(0,0,1 ; a, b, c)=\left(U_{n}\right)_{n \in \mathbb{Z}}$ be the sequences of polynomials defined by real numbers $p, q, r, a, b, c$ where $c \neq 0$. The determinant of the matrix $\left[1 / P_{s+k(n+i+j)}\right]_{0 \leq i, j \leq m}$ is given by

$$
\operatorname{det}\left(\left[\frac{1}{P_{s+k(n+i+j)}}\right]_{0 \leq i, j \leq m}\right)=\frac{c^{(s+k n)\left(\begin{array}{c}
m+1 \\
2
\end{array}\right)+2 k\left(\begin{array}{c}
m+1 \\
3
\end{array}\right)} \Delta_{\mathcal{P}}^{\left(\begin{array}{c}
m+1 \\
2
\end{array}\right)} \prod_{i=0}^{m} U_{k(i+1)}^{2(m-i)}}{(-1)^{(s+k n)\left(\begin{array}{c}
m+1 \\
2
\end{array}\right)} \prod_{0 \leq i, j \leq m} P_{s+k(n+i+j)}}
$$

where $\Delta_{\mathcal{P}}$ is the discriminant of $\mathcal{P}(x)(p, q, r ; a, b, c)$, provided that the denominators on both sides of the identity are nonzero.

Proof. The proof is essentially the same as the proof of Theorem 2 by applying (2.11), (2.12), (2.13) to Lemma 3 and some standard counting arguments.

Let $A$ be a $m \times m$ matrix. Let $A_{k}(i, j)$ be the determinant of the $k \times k$ submatrix of $A$ whose first entry is at the position of the $i^{t h}$-row and the $j^{t h}$-column of $A$.

Lemma 4. Let $A$ be a $m \times m$ matrix whose entries are rational functions in variable $x$. Then

$$
A_{m}(1,1) A_{m-2}(2,2)=A_{m-1}(1,1) A_{m-1}(2,2)-A_{m-1}(2,1) A_{m-1}(1,2) .
$$

Proof. We invoke the Desnanot-Jacobi identity [3] for a matrix $A$ of size $m \times m$ with indeterminates as entries.

$$
A_{m}(1,1) A_{m-2}(2,2)=A_{m-1}(1,1) A_{m-1}(2,2)-A_{m-1}(2,1) A_{m-1}(1,2) .
$$

To extend this result to the case where the matrix $A$ has rational functions as entries, we simply use the same strategy as in the proof of Lemma 1. 
Let $m \geq 1$. The rising powers of a sequence of polynomials $\mathcal{P}(x)(p, q, r ; a, b, c)=\left(P_{n}\right)_{n \in \mathbb{Z}}$ is denoted by $P_{n}^{\langle m\rangle}$, which is defined by

$$
P_{n}^{\langle m\rangle}:=P_{n} P_{n+1} \cdots P_{n+m-1} \text {. }
$$

Theorem 5. Let $n$ be any integer. Let $m \geq 1$ and $d \geq 1$. Let $\mathcal{P}(x)(p, q, r ; a, b, c)=\left(P_{n}\right)_{n \in \mathbb{Z}}$ and $U(x)(0,0,1 ; a, b, c)=\left(U_{n}\right)_{n \in \mathbb{Z}}$ be the sequences of polynomials defined by real numbers $p, q, r, a, b, c$ where $c \neq 0$. Then

$$
\begin{aligned}
& \operatorname{det}\left(\left[P_{n+i+j}^{\langle m\rangle}\right]_{0 \leq i, j \leq d-1}\right) \\
& =(-1)^{n\left(\begin{array}{c}
d \\
2
\end{array}\right)+\left(\begin{array}{c}
d+1 \\
3
\end{array}\right)} c^{(n+d-2)\left(\begin{array}{c}
d \\
2
\end{array}\right)} \Delta_{\mathcal{P}}^{\left(\begin{array}{c}
d \\
2
\end{array}\right)} \cdot \prod_{i=1}^{d-1}\left(U_{i} U_{r+1-i}\right)^{d-i} \cdot \prod_{i=d-1}^{2(d-1)} P_{n+1}^{\langle m+1-d\rangle}
\end{aligned}
$$

where $\Delta_{\mathcal{P}}$ is the discriminant of $\mathcal{P}(x)(p, q, r ; a, b, c)$.

Proof. The proof is based on induction on $d$, Lemma 4 and Theorem 1. It is essentially identical to the proof of Theorem 2.1 in the work of Tangboonduangjit and Thanatipanonda [11] and hence we skip it.

If we set $p=q=b=0$ and $r=a=c=1$, then we get the sequence of Fibonacci polynomials in $\mathcal{P}(x)(0,0,1 ; 1,0,1)=\left(F_{n}(x)\right)_{n \in \mathbb{Z}}$ where the sequence $\left(F_{n}(x)\right)_{n \in \mathbb{Z}}$ is defined by

$$
F_{0}(x) \equiv 0, \quad F_{1}(x) \equiv 1, \quad F_{n+2}(x)=x F_{n+1}(x)+F_{n}(x) .
$$

We recover the Fibonacci numbers and Pell numbers by evaluating $F_{n}(x)$ at $x=1$ and $x=2$ respectively. We note that $\Delta_{\mathcal{P}}=1$ and $\mathcal{U}(x)(0,0,1 ; 1,0,1)=\left(F_{n}(x)\right)_{n \in \mathbb{Z}}$. By Theorem 2, Theorem 3, Theorem 4 and Theorem 5, we get the following corollary:

Corollary 2. Let $m \geq 1$ and $d \geq 1$. Let $s, k, n$ be any integers. Let $\left(d_{i}\right)_{1 \leq i \leq m}$ and $\left(e_{i}\right)_{1 \leq i \leq m}$ be sequences of integers. Then

$$
\begin{aligned}
& \operatorname{det}\left(\left[\left(F_{s+k(n+i+j)}(x)\right)^{m}\right]_{0 \leq i, j \leq m}\right)=(-1)^{(s+k n+1)\left(\begin{array}{c}
m+1 \\
2
\end{array}\right) .} \\
& \cdot \prod_{i=0}^{m}\left(\begin{array}{c}
m \\
i
\end{array}\right)\left(F_{(i+1) k}(x)\right)^{2(m-i)}, \\
& \operatorname{det}\left(\left[\prod_{f=j+1}^{m} F_{s+k\left(n+i+d_{f}\right)}(x) \prod_{g=1}^{j} F_{s+k\left(n+i+e_{g}\right)}(x)\right]_{0 \leq i, j \leq m}\right) \\
& =(-1)^{(s+k n+1)\left(\begin{array}{c}
m+1 \\
2
\end{array}\right)+k\left(\begin{array}{c}
m+1 \\
3
\end{array}\right) \prod_{l=1}^{m}\left(F_{k l}(x)\right)^{m+1-l} \cdot \prod_{1 \leq i \leq j \leq m}(-1)^{k d_{j}} F_{k\left(e_{i}-d_{j}\right)}(x),}
\end{aligned}
$$




$$
\begin{aligned}
& \operatorname{det}\left(\left[\frac{1}{F_{s+k(n+i+j)}(x)}\right]_{0 \leq i, j \leq m}\right)=\frac{(-1)^{(s+k n)\left(\begin{array}{c}
m+1 \\
2
\end{array}\right)} \prod_{i=0}^{m}\left(F_{k(i+1)}(x)\right)^{2(m-i)}}{\prod_{0 \leq i, j \leq m} F_{s+k(n+i+j)}(x)}, \\
& \operatorname{det}\left(\left[\left(F_{n+i+j}(x)\right)^{\langle m\rangle}\right]_{0 \leq i, j \leq d-1}\right)
\end{aligned}
$$

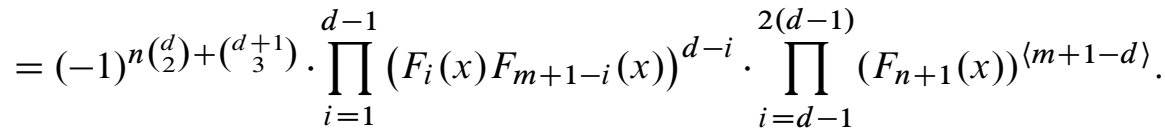

Remark 3. We recover the identities (1.2) and (1.4) by setting $x=1$ in the first identity and the last identity in Corollary 2 respectively.

Remark 4. We recover the results shown by Alfred [2] by setting $x=1, s=0, k=$ $1, n=0$ and $d_{i} \equiv 0, e_{i} \equiv 1$ for all $1 \leq i, j \leq m$ in the second identity in Corollary 2 .

Remark 5. We get the identity (1.5) by setting $x=1, s=0, k=n=1$ in the third identity in Corollary 2.

If we set $p=2, q=a=c=1$ and $r=b=0$, then we get the sequence of Lucas polynomials in $\mathcal{P}(x)(2,1,0 ; 1,0,1)=\left(L_{n}(x)\right)_{n \in \mathbb{Z}}$ where the sequence $\left(L_{n}(x)\right)_{n \in \mathbb{Z}}$ is defined by

$$
L_{0}(x) \equiv 2, \quad L_{1}(x) \equiv x, \quad L_{n+2}(x)=x L_{n+1}(x)+L_{n}(x) .
$$

We recover the Lucas numbers by evaluating $L_{n}(x)$ at $x=1$. We note that $\Delta_{\mathcal{P}}=$ $\left(-x^{2}-4\right)$ and $\mathcal{U}(x)(0,0,1 ; 1,0,1)=\left(F_{n}(x)\right)_{n \in \mathbb{Z}}$. By Theorem 2, Theorem 3, Theorem 4 and Theorem 5, we get the following corollary:

Corollary 3. Let $m \geq 1$ and $d \geq 1$. Let $s, k, n$ be any integers. Let $\left(d_{i}\right)_{1 \leq i \leq m}$ and $\left(e_{i}\right)_{1 \leq i \leq m}$ be sequences of integers.

$$
\begin{aligned}
& \operatorname{det}\left(\left[\left(L_{s+k(n+i+j)}(x)\right)^{m}\right]_{0 \leq i, j \leq m}\right) \\
& =(-1)^{\left.(s+k n)\left(_{2}^{m+1}\right)\left(x^{2}+4\right)^{(m+1}\right)} \prod_{i=0}^{m}\left(\begin{array}{c}
m \\
i
\end{array}\right)\left(F_{(i+1) k}(x)\right)^{2(m-i)} \text {, } \\
& \operatorname{det}\left(\left[\prod_{f=j+1}^{m} L_{s+k\left(n+i+d_{f}\right)}(x) \prod_{g=1}^{j} L_{s+k\left(n+i+e_{g}\right)}(x)\right]_{0 \leq i, j \leq m}\right)
\end{aligned}
$$

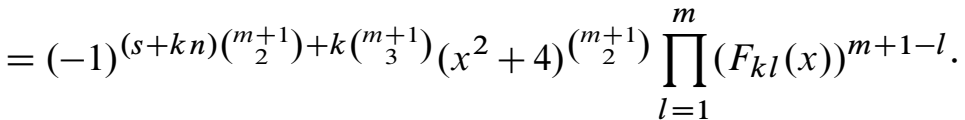

$$
\begin{aligned}
& \cdot \prod_{1 \leq i \leq j \leq m}(-1)^{k d_{j}} F_{k\left(e_{i}-d_{j}\right)}(x), \\
& \operatorname{det}\left(\left[\frac{1}{L_{s+k(n+i+j)}(x)}\right]_{0 \leq i, j \leq m}\right)
\end{aligned}
$$




$$
\begin{aligned}
& =\frac{(-1)^{(s+k n+1)\left(\begin{array}{c}
m+1 \\
2
\end{array}\right)\left(x^{2}+4\right)^{\left(\begin{array}{c}
m+1 \\
2
\end{array}\right)}}}{\prod_{0 \leq i, j \leq m} L_{s+k(n+i+j)}(x)} \prod_{i=0}^{m}\left(F_{k(i+1)}(x)\right)^{2(m-i)} \\
& \operatorname{det}\left(\left[\left(L_{n+i+j}(x)\right)^{\langle m\rangle}\right]_{0 \leq i, j \leq d-1}\right) \\
& =(-1)^{(n+1)\left(\begin{array}{c}
d \\
2
\end{array}\right)+\left(\begin{array}{c}
d+1 \\
3
\end{array}\right)}\left(x^{2}+4\right)^{\left(\begin{array}{c}
d \\
2
\end{array}\right)} \cdot \prod_{i=1}^{d-1}\left(F_{i}(x) F_{m+1-i}(x)\right)^{d-i} . \\
& \cdot \prod_{i=d-1}^{2(d-1)}\left(L_{n+1}(x)\right)^{\langle m+1-d\rangle} .
\end{aligned}
$$

If we set $p=q=1, a=2, c=-1$ and $r=b=0$, then we get the sequence of Chebyshev polynomials of the first kind in $\mathcal{P}(x)(1,1,0 ; 2,0,-1)=\left(T_{n}(x)\right)_{n \in \mathbb{Z}}$ where the sequence $\left(T_{n}(x)\right)_{n \in \mathbb{Z}}$ is defined by

$$
T_{0}(x) \equiv 1, \quad T_{1}(x) \equiv x, \quad T_{n+2}(x)=2 x T_{n+1}(x)-T_{n}(x) .
$$

We note that $\Delta_{\mathcal{P}}=\left(-x^{2}+1\right)$.

If we set $p=1, a=q=2, c=-1$ and $r=b=0$, then we get the sequence of Chebyshev polynomials of the second kind in $\mathcal{P}(x)(1,2,0 ; 2,0,-1)=\left(S_{n}(x)\right)_{n \in \mathbb{Z}}$ where the sequence $\left(S_{n}(x)\right)_{n \in \mathbb{Z}}$ is defined by

$$
S_{0}(x) \equiv 1, \quad S_{1}(x) \equiv 2 x, \quad S_{n+2}(x)=2 x S_{n+1}(x)-S_{n}(x) .
$$

We note that $\Delta_{\mathcal{P}}=\left(-2 x^{2}+1\right)$.

We note that

$$
U(x)(0,0,1 ; 2,0,-1)=\left(U_{n}(x)\right)_{n \in \mathbb{Z}}
$$

where

$$
U_{n}(x)=S_{n-1}(x) \text { for } n \in \mathbb{Z} .
$$

We get two corollaries by Theorem 2, Theorem 3, Theorem 4 and Theorem 5.

Corollary 4. Let $m \geq 1$ and $d \geq 1$. Let $s, k, n$ be any integers. Let $\left(d_{i}\right)_{1 \leq i \leq m}$ and $\left(e_{i}\right)_{1 \leq i \leq m}$ be sequences of integers. Then

$$
\begin{aligned}
& \operatorname{det}\left(\left[\left(T_{s+k(n+i+j)}(x)\right)^{m}\right]_{0 \leq i, j \leq m}\right)=\left(x^{2}-1\right)^{\left(\begin{array}{c}
m+1 \\
2
\end{array}\right)} \prod_{i=0}^{m}\left(\begin{array}{c}
m \\
i
\end{array}\right)\left(S_{(i+1) k-1}(x)\right)^{2(m-i)}, \\
& \operatorname{det}\left(\left[\prod_{f=j+1}^{m} T_{s+k\left(n+i+d_{f}\right)}(x) \prod_{g=1}^{j} T_{s+k\left(n+i+e_{g}\right)}(x)\right]_{0 \leq i, j \leq m}\right) \\
& =\left(x^{2}-1\right)^{\left(\begin{array}{c}
m+1 \\
2
\end{array}\right)} \prod_{l=1}^{m}\left(S_{k l-1}(x)\right)^{m+1-l} \prod_{1 \leq i \leq j \leq m} S_{k\left(e_{i}-d_{j}\right)-1}(x),
\end{aligned}
$$




$$
\begin{aligned}
& \operatorname{det}\left(\left[\frac{1}{T_{s+k(n+i+j)}(x)}\right]_{0 \leq i, j \leq m}\right)=\frac{\left(-x^{2}+1\right)^{\left(\begin{array}{c}
m+1 \\
2
\end{array}\right)} \prod_{i=0}^{m}\left(S_{k(i+1)-1}(x)\right)^{2(m-i)}}{\prod_{0 \leq i, j \leq m} T_{s+k(n+i+j)}(x)}, \\
& \operatorname{det}\left(\left[\left(T_{n+i+j}(x)\right)^{\langle m\rangle}\right]_{0 \leq i, j \leq d-1}\right) \\
& =(-1)^{d\left(\begin{array}{c}
d \\
2
\end{array}\right)+\left(\begin{array}{c}
d+1 \\
3
\end{array}\right)}\left(-x^{2}+1\right)^{\left(\begin{array}{c}
d \\
2
\end{array}\right)} \prod_{i=1}^{d-1}\left(S_{i-1}(x) S_{m-i}(x)\right)^{d-i} \cdot \prod_{i=d-1}^{2(d-1)}\left(T_{n+1}(x)\right)^{\langle m+1-d\rangle} .
\end{aligned}
$$

Corollary 5. Let $m \geq 1$ and $d \geq 1$. Let $s, k, n$ be any integers. Let $\left(d_{i}\right)_{1 \leq i \leq m}$ and $\left(e_{i}\right)_{1 \leq i \leq m}$ be sequences of integers. Then

$$
\begin{aligned}
& \operatorname{det}\left(\left[\left(S_{S+k(n+i+j)}(x)\right)^{m}\right]_{0 \leq i, j \leq m}\right)=\left(2 x^{2}-1\right)^{\left(\begin{array}{c}
m+1 \\
2
\end{array}\right)} \cdot \prod_{i=0}^{m}\left(\begin{array}{c}
m \\
i
\end{array}\right)\left(S_{(i+1) k-1}(x)\right)^{2(m-i)}, \\
& \operatorname{det}\left(\left[\prod_{f=j+1}^{m} S_{s+k\left(n+i+d_{f}\right)}(x) \prod_{g=1}^{j} S_{s+k\left(n+i+e_{g}\right)}(x)\right]_{0 \leq i, j \leq m}\right) \\
& =\left(2 x^{2}-1\right)^{\left(\begin{array}{c}
m+1 \\
2
\end{array}\right)} \prod_{l=1}^{m}\left(S_{k l-1}(x)\right)^{m+1-l} \prod_{1 \leq i \leq j \leq m} S_{k\left(e_{i}-d_{j}\right)-1}(x), \\
& \operatorname{det}\left(\left[\frac{1}{S_{S+k(n+i+j)}(x)}\right]_{0 \leq i, j \leq m}\right)=\frac{\left(-2 x^{2}+1\right)^{(m+1)} \prod_{i=0}^{m}\left(S_{k(i+1)-1}(x)\right)^{2(m-i)}}{\prod_{0 \leq i, j \leq m} S_{s+k(n+i+j)}(x)}, \\
& \operatorname{det}\left(\left[\left(S_{n+i+j}(x)\right)^{\langle m\rangle}\right]_{0 \leq i, j \leq d-1}\right) \\
& =(-1)^{d\left(\begin{array}{c}
d \\
2
\end{array}\right)+\left(\begin{array}{c}
d+1 \\
3
\end{array}\right)}\left(-2 x^{2}+1\right)^{\left(\begin{array}{c}
d \\
2
\end{array}\right)} \prod_{i=1}^{d-1}\left(S_{i-1}(x) S_{m-i}(x)\right)^{d-i} \cdot \prod_{i=d-1}^{2(d-1)}\left(S_{n+1}(x)\right)^{\langle m+1-d\rangle} .
\end{aligned}
$$

By Favard's theorem [6] (see also the standard reference textbook by Chihara [5, Chapter 2]), the sequence $\mathcal{P}(x)(1, q, r ; 1, b, c)=\left(P_{n}\right)_{n \in \mathbb{Z}_{\geq 0}}$ forms a sequence of orthogonal polynomials (with respect to certain linear functional) for $q \neq 0$ and $c \neq 0$. By Theorem 2, Theorem 3, Theorem 4 and Theorem 5, we state some determinant identities for matrices containing (powers of) such orthogonal polynomials.

Corollary 6. Let $n \geq 0, m \geq 1$ and $d \geq 1$. Let $\left(P_{n}\right)_{n \in \mathbb{Z}_{\geq 0}}$ be a sequence of orthogonal polynomials of the form:

$$
P_{0} \equiv 1, \quad P_{1} \equiv q x+r, \quad P_{n+2}=(x+b) P_{n+1}+c P_{n}
$$

where $c \neq 0, q \neq 0$ and $r, b$ are any real numbers. Then

$$
\begin{aligned}
& \operatorname{det}\left(\left[P_{n+i+j}^{m}\right]_{0 \leq i, j \leq m}\right) \\
& =(-1)^{(n+1)\left(\begin{array}{c}
m+1 \\
2
\end{array}\right)} \cdot \Delta^{\left(\begin{array}{c}
m+1 \\
2
\end{array}\right)} \cdot c^{n\left(\begin{array}{c}
m+1 \\
2
\end{array}\right)+2\left(\begin{array}{c}
m+1 \\
3
\end{array}\right)} \cdot \prod_{i=0}^{m}\left(\left(\begin{array}{c}
m \\
i
\end{array}\right) U_{i+1}^{2(m-i)}\right),
\end{aligned}
$$




$$
\begin{aligned}
& \operatorname{det}\left(\left[\prod_{f=j+1}^{m} P_{n+i+d_{f}} \prod_{g=1}^{j} P_{n+i+e_{g}}\right]_{0 \leq i, j \leq m}\right) \\
& =(-\Delta)^{\left(\begin{array}{c}
m+1 \\
2
\end{array}\right)}(-c)^{n\left(\begin{array}{c}
m+1 \\
2
\end{array}\right)+\left(\begin{array}{c}
m+1 \\
3
\end{array}\right)} \prod_{l=1}^{m} U_{l}^{m+1-l} \cdot \prod_{1 \leq i \leq j \leq m}(-c)^{d_{j}} U_{e_{i}-d_{j}}, \\
& \operatorname{det}\left(\left[\frac{1}{P_{s+k(n+i+j)}}\right]_{0 \leq i, j \leq m}\right)
\end{aligned}
$$

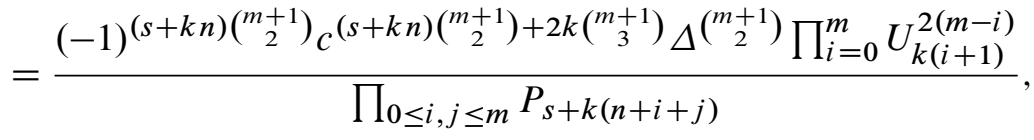

$$
\begin{aligned}
& \operatorname{det}\left(\left[P_{n+i+j}^{\langle m\rangle}\right]_{0 \leq i, j \leq d-1}\right) \\
& =(-1)^{n\left(\begin{array}{c}
d \\
2
\end{array}\right)+\left(\begin{array}{c}
d+1 \\
3
\end{array}\right)} c^{(n+d-2)\left(\begin{array}{c}
d \\
2
\end{array}\right)} \Delta^{\left(\begin{array}{c}
d \\
2
\end{array}\right)} \cdot \prod_{i=1}^{d-1}\left(U_{i} U_{m+1-i}\right)^{d-i} \cdot \prod_{i=d-1}^{2(d-1)} P_{n+1}^{\langle m+1-d\rangle} .
\end{aligned}
$$

where $\Delta=\left(q^{2}-q\right) x^{2}+(q r-r-b q) x+\left(r^{2}-b r-c\right)$ and $\left(U_{n}\right)_{n \in \mathbb{Z}_{\geq 0}}$ is the sequence of orthogonal polynomials defined by

$$
U_{0} \equiv 0, \quad U_{1} \equiv 1, \quad U_{n+2}=(x+b) U_{n+1}+c U_{n} .
$$

\section{ACKNOWLEDGEMENT}

The author expresses his gratitude to the Mahidol University International College (MUIC) at Bangkok, Thailand for their hospitality in hosting me in May 2018. The author is grateful to Thotsaporn Thanatipanonda for generously sharing his ideas on the topic. The author is supported by Startup Grant 2016 (G00002235) which is funded by United Arab Emirates University.

\section{REFERENCES}

[1] B. U. Alfred, “ Advanced problems and solutions ," Fibonacci Quart., vol. 1, no. 1, p. 48, 1963.

[2] B. U. Alfred, "Some determinants involving powers of Fibonacci numbers. ," Fibonacci Quart., vol. 2, no. 2, pp. 81-92, 1964.

[3] T. Amdeberhan and D. Zeilberger, "Determinants through the looking glass." Adv. in Appl. Math., vol. 27, no. 2-3, pp. 225-230, 2001, doi: 10.1006/aama.2001.0732.

[4] L. Carlitz, "Some determinants containing powers of Fibonacci numbers. ," Fibonacci Quart. , vol. 4, no. 2, pp. 129-134, 1966.

[5] T. S. Chihara, An Introduction to Orthogonal Polynomials. New York: Gordon and Breach Science Publishers, 1978.

[6] J. Favard, "Sur les polynomes de Tchebicheff." C. R. Acad. Sci., vol. 200, pp. 2052-2053, 1935.

[7] C. Krattenthaler, "Advanced determinant calculus: a complement." Linear Algebra Appl., vol. 411, pp. 68-166, 2005, doi: 10.1016/j.1aa.2005.06.042.

[8] R. S. Melham and A. G. Shannon, "A generalization of the Catalan identity and some consequences." Fibonacci Quart., vol. 33, no. 2, pp. 82-84, 1995. 
[9] F. D. Parker, "Advanced problems and solutions," Fibonacci Quart., vol. 2, no. 4, p. 303, 1964.

[10] H. Prodinger, "Determinants containing rising powers of Fibonacci numbers." Fibonacci Quart., vol. 54, no. 2, pp. 137-141, 2016.

[11] A. Tangboonduangjit and T. Thanatipanonda, " Determinants of rising powers of second order linear recurrence entries by means of the Desnanot-Jacobi identity. ," Fibonacci Quart., vol. 54, no. 4, pp. 340-343, 2016

[12] A. Tangboonduangjit and T. Thanatipanonda, "Determinants containing powers of generalized Fibonacci numbers." J. Integer Seq., vol. 19, no. 7, p. 9 pp., 2016.

Author's address

\section{H.-H. Leung}

United Arab Emirates University, Department of Mathematical Sciences, Al Ain, 15551, United Arab Emirates

E-mail address: hohon. leung@uaeu.ac.ae 